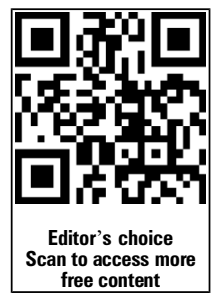

- Additional material is published online only. To view please visit the journal online (http://dx.doi.org/10.1136/ bmjspcare-2013-000604).

For numbered affiliations see end of article.

\section{Correspondence to}

Dr Keith G Wilson, Department of Psychology, The Ottawa Hospital Rehabilitation Centre, 505 Smyth Road, Ottawa, ON, Canada, K1H 8M2; Kewilson@toh.on.ca

Received 15 October 2013 Revised 16 January 2014 Accepted 13 February 2014 Published Online First 4 March 2014
CrossMark

To cite: Wilson $\mathrm{KG}$ Dalgleish TL, Chochinov HM, et al. BMJ Supportive \& Palliative Care 2016;6: 170-177.

\title{
Mental disorders and the desire for death in patients receiving palliative care for cancer
}

\author{
Keith G Wilson, ${ }^{1}$ Tracy L Dalgleish, ${ }^{2}$ Harvey Max Chochinov, ${ }^{3}$ Srini Chary, ${ }^{4}$ \\ Pierre R Gagnon, ${ }^{5}$ Karen Macmillan, ${ }^{6}$ Marina De Luca, ${ }^{7}$ Fiona O'Shea, ${ }^{8}$ \\ David Kuhl, ${ }^{9}$ Robin L Fainsinger ${ }^{10}$
}

\begin{abstract}
Objectives The desire for death in terminally ill patients is associated with depression and anxiety, but not all patients who report it meet criteria for mental disorders. We examined the characteristics of subgroups of palliative cancer patients who expressed a desire for death that occurred either with or without a concurrent depressive or anxiety disorder.

Design Cross-sectional survey.

Setting Eight Canadian palliative care programs.

Participants 377 patients with cancer.
\end{abstract}

Main outcome measures Desire for Death Rating

Scale; Structured Interview of Symptoms and

Concerns.

Results Most participants (69.5\%) had no desire for death. Of the remainder, 69 (18.3\%)

acknowledged occasional transient thoughts, and $46(12.2 \%)$ reported an apparently genuine desire to die. In the latter group, 24 individuals (52.2\%) were diagnosed with a mental disorder and 22 (44.8\%) were not. Individuals with no serious desire for death and no mental disorder reported the least distress in physical, social, existential, and psychological symptoms and concerns; those with a mental disorder and a significant desire for death reported the most. The subgroup of patients with a serious desire for death but no concurrent mental disorders still reported increased distress due to physical symptoms and social concerns, as well as a higher prevalence of global suffering.

Conclusions The expression of a desire for death by a terminally ill patient should raise a suspicion about mental health problems, but is not in itself clearly indicative of one. Nevertheless, it may serve as a catalyst to review the individual's physical symptom management and interpersonal concerns, and overall sense of suffering.

\section{INTRODUCTION}

The societal interest in the euthanasia/ assisted-suicide debate has encouraged research into the prevalence and correlates of the desire for death in the terminally ill. This research has shown that the desire for death is actually quite common among patients with advanced, life-threatening illnesses. ${ }^{1}$ In palliative care settings, it has been found that $11-55 \% \%^{2-9}$ of patients experience such a desire at least transiently, and from 3-20\% ${ }^{2-6}{ }^{8-12}$ report a more pervasive and apparently sincere wish to die.

Although there have been inconsistencies, various studies have reported low to moderate correlations between the desire for death and pain, ${ }^{3} 7$ 13-15 fatigue and other physical symptoms, ${ }^{7-11}$ weak social support, ${ }^{3} 4{ }^{4} 711$ diminished functional status, ${ }^{9} 1516$ low quality of life, ${ }^{2} 915$ anxiety $^{11} 1215$ and hopelessness. ${ }^{2} 8914$ 16-19 Perhaps the most well-replicated correlation, however, has been with measures of depres-

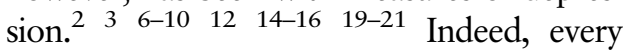
study that has looked for an association between depression and the desire for death has found one, and Brown et $a l^{22}$ reported that all such patients were clinically depressed.

Nevertheless, research based on structured diagnostic interviews has reached the more tempered conclusion that mental disorders are evident among many, but not all, terminally ill patients who express a desire for death. In most studies, the prevalence of diagnosed disorders among these individuals has ranged from 47$80 \% .^{2} 36 \quad 8 \quad 1019$ This suggests that the desire for death is often embedded in a broader context of clinically significant psychological distress, but not always. Qualitative studies have confirmed that there may be different pathways by which individuals arrive at a desire for death. Nissim et $a l^{23}$ reported that a hastened death is sometimes seen as a hypothetical 
exit strategy that could forestall an anticipated experience of suffering. For some patients, however, this desire is not hypothetical at all, but real and immediate. Even in these cases, Nissim et $\mathrm{al}^{23}$ identified a dichotomy; for some, the desire for death is an expression of despair, whereas for others it is a manifestation of letting go. In the present study, we hypothesize that the former pathway may be more characteristic of those patients who also have significant psychological disorders, whereas the latter pathway may be more likely among those who are not necessarily clinically anxious or depressed, but who have reached a stage of illness at which they are ready for death to come. Our main goal, therefore, is to examine the characteristics of patients with advanced cancer whose desire for death is embedded in these two different pathways: either with or without a concurrently diagnosed mental disorder.

\section{METHODS}

\section{Study design}

This project is a secondary analysis of data from the Canadian National Palliative Care Survey (NPCS), a multicentre, cross-sectional study of patients receiving palliative care for cancer. NPCS interviews were conducted between May 2001 and March 2003.

\section{Sample}

A detailed description of the recruitment procedures and demographic characteristics of the NPCS participants are reported in previous publications. ${ }^{24}{ }^{25}$ Participants were recruited from among consecutive admissions to inpatient palliative care units, hospital consultation services, or home care. Inclusion criteria included that: (1) the patient had a diagnosis of cancer and had been informed of the prognosis; (2) a palliative care clinician estimated that survival duration was under 6 months; (3) the patient was deemed to be cognitively competent and medically stable enough to participate in the interview and (4) the patient could converse in English or French.

A total of 377 patients provided complete information regarding the desire for death and details relevant to the diagnosis of depression and anxiety disorders. An earlier NPCS report addressed the overall prevalence of mental disorders. ${ }^{26}$ As yet, however, there has not been an explicit focus on the co-occurrence of these disorders with the desire for death.

\section{Procedure}

Patients were interviewed by professional staff who conducted face-to-face, semistructured interviews. The interviewers all had previous clinical experience in palliative care nursing, psychology, social work, or education. They were trained in the specific interview protocol at a central two-day workshop before recruitment began.

\section{Ethics}

The protocol was approved by the research ethics boards of all institutions involved, including: Memorial
University of Newfoundland Human Investigation Committee; Centre hospitalier universitaire de Québec, comité d'éthique de la recherche; The Ottawa Hospital Research Ethics Board; SCO Health Service Research Ethics Board; The Ottawa Institute for Rehabilitation Research and Development Research Ethics Committee; University of Manitoba Health Research Ethics Board; University of Saskatchewan Advisory Committee on Ethics in Behavioural Science Research; Alberta Cancer Board Research Ethics Committee; University of Alberta Health Research Ethics Board (B: Health Research); Caritas Health Group Research Steering Committee; British Columbia Cancer Agency Research Ethics Board; Kelowna General Hospital Institutional Research Review Committee; University of British Columbia Providence Health Care Research Ethics Board. All patients provided written informed consent prior to participation.

\section{Measures}

Demographic and social characteristics

The interview began by reviewing basic demographic characteristics. It then proceeded to cover a range of issues associated with the individual's experience of various physical and psychological problems.

\section{Structured Interview of Symptoms and Concerns}

The Structured Interview of Symptoms and Concerns $(\mathrm{SISC})^{27}$ assesses end-of-life issues through the use of single-item screening that covers common physical symptoms (six items: general malaise, pain, drowsiness, nausea, weakness, breathlessness), social concerns (four items: social isolation, interpersonal communication problems, self-perceived burden to others, financial difficulties), existential issues (six items: spiritual crisis, difficulty accepting, general dissatisfaction with life, loss of dignity, loss of resilience, loss of control), and mentalhealth problems (four items: anxiety, depressed mood, loss of interest or pleasure in activities, and hopelessness). Two additional items assess the overall sense of suffering and the desire for death.

Each item on the SISC begins with a structured lead question into the presence of a particular symptom or concern. This is followed by questions probing into the severity of the issue, which is then rated by the interviewer on a seven-point scale (anchored at $0=$ no problem and $6=$ an extremely severe problem). For each symptom or concern, a score $=3$ represents a 'moderate' level of severity, and is the threshold at which the symptom or concern has been identified by the participant as a significant problem. This threshold has been used in previous research to identify the prevalence of clinically significant problems in particular areas. ${ }^{3} 252829$

Depression and anxiety disorders

Depression and anxiety disorders were assessed with the SISC mental health questions in conjunction with items drawn from the Primary Care Evaluation of Mental Disorders clinician interview guide (PRIME-MD). ${ }^{30}$ The PRIME-MD is based on the diagnostic criteria 
outlined in the Diagnostic and Statistical Manual of Mental Disorders, fourth edition (DSM-IV), ${ }^{31}$ and provides a quick screening method for a range of discrete diagnoses. The present modification involved the substitution of the original PRIME-MD screening questions for depressed or hopeless mood, loss of interest or pleasure in activities, and anxiety, with corresponding items from the SISC. The SISC approach provides a more in-depth discussion of these issues, and ensures that the symptom ratings adhere to severity thresholds specified in the DSM-IV. ${ }^{32}$ The remaining symptom criteria for each disorder were retained from the PRIME-MD interview, which allowed for the diagnosis of major depression, major depression in partial remission, minor depression, dysthymia, panic disorder, generalised anxiety disorder, anxiety disorder not otherwise specified and anxiety disorder secondary to a general medical condition. ${ }^{26}$

Desire for death

The SISC lead question for the desire for death asked, 'Do you ever wish that your illness would progress more quickly so that your suffering could be over sooner?' If the participant responded affirmatively, follow-up questions included: 'Do you ever wish for death?' 'How often do you feel this way?' 'Is it quite constant, or are there times when you do not feel that way?' 'Do you pray for death to come soon?'

The lead and follow-up questions were derived from those reported by Chochinov et al. ${ }^{3}$ As with other SISC items, the desire for death was rated on a 0 (no desire for death) to 6-point (extreme) scale, with a rating of $3=$ 'moderate' corresponding to the respondent's having 'a genuine desire for early death that is reported to be consistent over time, but he/she is not consumed with the prospect'. This cut-off has been used in previous research to identify individuals with a 'serious and pervasive' desire to die. ${ }^{2} 568101119$

\section{Analysis}

SPSS statistical software package (V.18) was used for all analyses. Many of the distributions for individual SISC items, including the desire for death, were positively skewed, indicating that most participants reported minimal difficulties in those areas. For some analyses, therefore, the SISC scores are categorised with regard to whether the participant indicated that the symptom or concern was a significant problem (ie, scores $\geq 3$ ). The SISC data for these individual symptoms and concerns were then analysed using a nonparametric approach. In order to provide a broader perspective, however, we also computed subscale scores by summing the original item ratings within the a priori dimensions of physical symptoms, social concerns, existential issues and psychological problems. ${ }^{25}$

We first examined the prevalence of the desire for death in the entire sample at the different severity levels. Participants were then classified into two groups scoring either above or below the cut-off on the desire-for-death rating scale. Preliminary analyses were undertaken to compare these two groups, and are reported in online supplementary table S1.

Next, participants were divided further into subgroups of individuals who either did, or did not, meet DSM-IV diagnostic criteria for a depressive or anxiety disorder. These four groups were then compared, using analyses of variance for continuous variables and $\chi^{2}$ analyses for categorical.

\section{RESULTS}

\section{Sample characteristics}

The 377 participants included 169 males (44.8\%). The mean age of participants was 67.2 years $(\mathrm{SD}=12.9)$, and the median survival duration until death was 63 days. The majority of participants were Caucasian (94.4\%). There were 91 (24.1\%) participants who met criteria for an anxiety or depressive disorder.

\section{Prevalence of the desire for death}

The distribution of scores on the desire-for-death rating scale is shown in table 1 . Although the majority of participants $(69.5 \%)$ had no desire for death, there were $115(30.5 \%)$ individuals who experienced at least an occasional wish to die (scores $\geq 1$ ). Of this group, $46(12.2 \%$ of the entire sample) participants

Table 1 Distribution of scores on the desire-for-death rating scale $(n=377)$

\begin{tabular}{|c|c|c|c|}
\hline $\begin{array}{l}\text { Desire for death } \\
\text { score }\end{array}$ & Description & $\begin{array}{l}\text { Number of } \\
\text { patients }\end{array}$ & $\%$ \\
\hline 0 & No desire for death & 262 & 69.5 \\
\hline 1 & Minimal—eg, only occasionally has fleeting thoughts & 38 & 10.1 \\
\hline 2 & Mild_-eg, sometimes feels he/she would like an early death, but not always & 31 & 8.2 \\
\hline 3 & $\begin{array}{l}\text { Moderate-eg, has a genuine desire for early death that is reported to be consistent over time, but is } \\
\text { not consumed with the prospect }\end{array}$ & 11 & 2.9 \\
\hline 4 & Strong-eg, most of the time feels a pervasive wish for death to come soon & 14 & 3.7 \\
\hline 5 & Severe-eg, almost all of the time has a strong desire for death to come; prays for death & 9 & 2.4 \\
\hline 6 & $\begin{array}{l}\text { Extreme-eg, constant strong desire for death to come soon; thinks of little else; often prays for } \\
\text { death }\end{array}$ & 12 & 3.2 \\
\hline
\end{tabular}


received moderate-to-extreme scores, and are considered to have had a serious desire for death.

\section{Prevalence of mental disorders}

Psychological disorders were more common among those with a serious desire for death $(\mathrm{p}<0.001)$. Of the 46 participants in this group, $24(52.2 \%)$ met DSM-IV criteria for either depression $(\mathrm{n}=8)$, an anxiety disorder $(\mathrm{n}=1)$, or both $(\mathrm{n}=15)$, whereas 22 $(47.8 \%)$ did not. Of the participants with no significant desire for death $(\mathrm{n}=331), 67(20.2 \%)$ met criteria for a mental disorder and $264(79.8 \%)$ did not. Thus, the participants with a serious desire for death were about 2.5 times more likely to be diagnosed with a psychological disorder.

\section{Mental disorders and the desire for death}

We then examined the demographic characteristics and symptom profiles among four groups of participants who varied in the presence/absence of a DSM-IV disorder, as well as in the presence/absence of a serious desire for death.

There were no significant differences between the four groups in gender, marital status, living arrangement, education and median survival duration, ps $>0.19$. However, they differed with respect to age, $F$ $(3,373)=4.14, p=0.007$. Participants with a mental disorder but no significant desire for death $(\mathrm{M}=63.2$ years, $\mathrm{SD}=13.1)$ were younger than those with no disorder, either with a serious desire for death $(\mathrm{M}=72.6$ years, $\mathrm{SD}=11.3)$ or without $(\mathrm{M}=68.0$ years, $\mathrm{SD}=12.9)$, ps $<0.04$. Participants with a diagnosed disorder and a serious desire for death $(\mathrm{M}=65.0$ years, $\mathrm{SD}=11.0$ ) did not differ from any of the other groups.

The rates of problematic symptoms and concerns are shown in table 2. On the total subscale scores, there were overall differences on each of the physical, $\mathrm{F}(3,373)=17.95, \mathrm{p}<0.001$, social-relational, $\mathrm{F}(3,373)$ $=11.10, \mathrm{p}<0.001$, existential, $\mathrm{F}(3,373)=17.60$, and psychological, $\mathrm{F}(3,373)=88.15, \mathrm{p}<0.001$, dimensions. At the level of individual symptoms and concerns, there were group differences in 18 of 20 comparisons ( $p s<0.008$ ), as well as in the global of experience of suffering $(\mathrm{p}<0.001)$.

Pairwise posthoc comparisons were conducted to identify the specific contrasts that accounted for these overall effects. They revealed that individuals with no mental disorder and no significant desire for death had lower scores on all four SISC subscales than both groups of patients with mental disorders ( $\mathrm{ps}<0.001)$.

Not surprisingly, the two groups diagnosed with mental disorders also had more severe psychological symptoms than patients who reported a serious desire for death in the absence of a disorder $(p s<0.001)$. The latter group also had lower existential distress than those who had a desire for death and a mental disorder $(p<0.001)$, but they were similar in this regard to those who had a mental disorder alone $(p>0.10)$.
These three groups (ie, serious desire for death with a disorder, serious desire for death without a disorder, no serious desire for death with a disorder) did not differ from one another on the subscale scores for physical symptoms or social concerns ( $p s>0.094)$.

When the two groups with mental-health diagnoses were compared, those with a significant desire for death were found to have more severe difficulties in the existential and psychological domains ( $p s<0.001)$.

The last pairwise contrast involved comparisons of patients with or without a serious desire for death who did not meet diagnostic criteria for an anxiety or depressive disorder. This is an important comparison because it examines the profile of individuals who express a desire for death that is independent of a concurrent mental health problem. Indeed, these groups did not differ in their total score ratings for either psychological or existential issues ( $p s>0.34$ ). However, individuals with a serious desire for death did report more severe physical symptoms $(\mathrm{p}=0.01)$ and more distress around social concerns $(p<0.001)$. Of the individual problems, a greater percentage of those with a desire for death reported ongoing difficulty with the physical symptoms of general malaise, drowsiness, weakness and breathlessness ( $\mathrm{ps}<0.05$ ), as well as with the social concerns of isolation and self-perceived burden to others ( $\mathrm{ps}<0.01)$.

A final analysis was undertaken of the ratings on the single item that inquired about the participant's global level of suffering. The proportion of patients with high levels of suffering was greatest among the group with a concurrent mental disorder and a serious desire for death. Over $83 \%$ of these patients suffered to a clinically important degree, which was significantly greater than each of the other groups ( $p s<0.003)$. Those with neither a serious desire for death nor a mental disorder suffered the least $(13.6 \%$; ps<0.003). The remaining two groups had comparable overall rates of suffering (44.8\% for no serious desire for death and a mental disorder versus $40.9 \%$ for a serious desire for death and no mental disorder; $\mathrm{p}=0.43$ ).

\section{DISCUSSION}

In the first part of this study, we found that $30.5 \%$ of the participants experienced a desire for death at least transiently, with $12.2 \%$ reporting a more serious desire that was rated as an apparently genuine wish. Both prevalence estimates are within the ranges reported by previous investigators. ${ }^{2-12}$ We also found that $52 \%$ of those with a serious desire for death met diagnostic criteria for a depressive or anxiety disorder. As in previous research based on diagnostic interviews, ${ }^{2} 381019$ mental disorders were much more prevalent among those with a serious desire for death than those without. Therefore, the expression of a desire for death by a terminally ill patient should raise a suspicion about mental health problems; by itself, however, it is not definitively diagnostic of one. 
Table 2 Symptoms and concerns reported by participants with or without a serious desirefor death, broken down by the presence or absence of a mental disorder

\begin{tabular}{|c|c|c|c|c|c|}
\hline \multirow[b]{2}{*}{ Symptom or concern } & \multicolumn{2}{|c|}{ No serious desire for death $(n=331)$} & \multicolumn{2}{|c|}{ Serious desire for death $(n=46)$} & \multirow[b]{2}{*}{$\mathrm{p}$ Value } \\
\hline & No disorder $(n=264)$ & Disorder $(n=67)$ & No disorder $(\mathrm{n}=22)$ & Disorder $(n=24)$ & \\
\hline \multicolumn{6}{|l|}{ Physical problems } \\
\hline General malaise & $89(33.7)$ & $37(55.2)$ & $14(63.6)$ & $18(75.0)$ & $<0.001$ \\
\hline Drowsiness & $65(24.6)$ & $30(44.8)$ & $12(54.5)$ & $14(58.3)$ & $<0.001$ \\
\hline Nausea & $31(11.7)$ & $18(26.9)$ & $6(27.3)$ & $9(37.5)$ & $<0.001$ \\
\hline Weakness & $140(53.0)$ & $43(64.2)$ & $17(77.3)$ & $22(91.7)$ & $<0.001$ \\
\hline Breathlessness & $65(24.6)$ & $17(25.4)$ & $8(36.4)$ & $8(33.3)$ & 0.54 \\
\hline Pain & $74(28.0)$ & $33(49.3)$ & $7(31.8)$ & $13(54.2)$ & 0.001 \\
\hline Total (M, SD) & $9.3(5.6)$ & $13.2(6.7)$ & $13.3(6.1)$ & $16.4(5.1)$ & $<0.001$ \\
\hline \multicolumn{6}{|l|}{ Social-relational concerns } \\
\hline Social isolation & $12(4.5)$ & $17(25.4)$ & $7(31.8)$ & $8(33.3)$ & $<0.001$ \\
\hline Communication problem & $2(0.8)$ & $2(3.0)$ & $2(9.1)$ & $2(8.3)$ & 0.007 \\
\hline Burden to others & $53(20.1)$ & $24(35.8)$ & $10(45.5)$ & $11(45.8)$ & 0.001 \\
\hline Financial problem & $20(7.6)$ & $12(17.9)$ & $1(4.5)$ & $1(4.2)$ & 0.04 \\
\hline Total (M, SD) & $2.2(2.4)$ & $4.4(3.8)$ & $4.8(3.6)$ & $5.0(4.3)$ & $<0.001$ \\
\hline \multicolumn{6}{|l|}{ Existential issues } \\
\hline Loss of resilience $\ddagger$ & $19(3.8)$ & $8(12.1)$ & $2(9.1)$ & $13(54.2)$ & $<0.001$ \\
\hline Loss of dignity & $9(3.4)$ & $9(13.4)$ & $2(9.1)$ & $5(20.8)$ & 0.001 \\
\hline Loss of control & $5(1.9)$ & $9(13.4)$ & $3(13.6)$ & $8(33.3)$ & $<0.001$ \\
\hline Spiritual crisisł & $4(1.5)$ & $5(7.5)$ & $1(4.8)$ & $1(4.2)$ & 0.07 \\
\hline Difficulty accepting & $17(6.4)$ & $9(13.4)$ & $1(4.5)$ & $6(25.0)$ & 0.007 \\
\hline Dissatisfaction with life & $7(2.7)$ & $8(11.9)$ & $1(4.5)$ & $3(12.5)$ & 0.006 \\
\hline Total (M, SD) & $1.9(2.4)$ & $4.7(3.8)$ & $3.0(2.9)$ & $7.7(6.3)$ & $<0.001$ \\
\hline \multicolumn{6}{|l|}{ Psychological difficulties } \\
\hline Anxiety & $10(3.8)$ & $29(43.3)$ & $0(0.0)$ & $18(75.0)$ & $<0.001$ \\
\hline Depression & $7(2.7)$ & $29(43.3)$ & $0(0.0)$ & $16(66.7)$ & $<0.001$ \\
\hline Loss of interest/pleasure* & $10(3.8)$ & $27(40.9)$ & $2(9.1)$ & $14(58.3)$ & $<0.001$ \\
\hline Hopelessness $†$ & $4(1.5)$ & $21(31.3)$ & $3(13.6)$ & $14(58.3)$ & $<0.001$ \\
\hline Total (M, SD) & $1.9(1.9)$ & $7.8(3.9)$ & $2.9(2.4)$ & $11.5(4.6)$ & $<0.001$ \\
\hline \multicolumn{6}{|l|}{ Global considerations } \\
\hline Suffering & $36(13.6)$ & $30(44.8)$ & $9(40.9)$ & $20(83.3)$ & $<0.001$ \\
\hline
\end{tabular}

Table entries are $n(\%)$ of participants who endorsed specific symptoms or concerns at a moderate-to-extreme level; $p$ values are based on $\chi^{2}$ tests for individual symptoms and concerns and one-way analyses of variance for total scores. Total scores refer to the mean of the summed item ratings within each of the physical, social-relational, existential and psychological dimensions.

*One patient with missing data.

†Two patients with missing data.

In the terminology of Nissim et $a l,{ }^{23}$ we considered that the desire for death among patients with a concurrent mental disorder was more likely to represent an 'expression of despair' rather than a 'manifestation of letting go'. As such, it was possible that patients who arrived at the desire for death via this pathway would differ in other clinically relevant ways from patients with no concurrent mental health problem. In this context, there were several major findings. First, it was evident that patients who had neither a serious desire for death nor a mental disorder were experiencing the least problematic course of illness. They differed from patients with concurrent mental disorders in all dimensions of symptoms and concerns, and also experienced less physical and social distress than those with a serious desire for death in the absence of a disorder. Hence, this group appears to have been coping well and achieving optimal palliative care outcomes. Importantly, this was also the largest group in the study, comprising $70 \%$ of the participants.

The psychological items of the SISC provided the initial basis for screening for the core symptoms of the depressive and anxiety disorders. It was to be expected, therefore, that individuals diagnosed with these disorders would report greater psychological distress than those who were not, even if the latter group expressed a significant desire for death. This was indeed found. However, a second major finding was that patients with a serious desire for death and a concurrent mental disorder were experiencing the most difficult course of illness. The extent of their 
psychological and existential distress was greater than that of all other groups, including those with a mental disorder but no significant desire for death. They also had the most pervasive experience of suffering.

Apart from more severe psychological symptoms, patients with a mental disorder but no serious desire for death were similar to those with a desire for death but no mental disorder on the existential, social, and physical symptom dimensions. They also had similar rates of global suffering. They were younger, however. An association between younger age and psychological distress has been noted in other research, including previous reports from the NPCS. ${ }^{25} 262829$ It may be that younger individuals experience a more acute sense of untimely death that presents a risk for depression and anxiety, ${ }^{26}$ have less developed coping strategies for dealing with death, have more concern about the welfare of dependants, or are simply more willing to disclose psychological problems. ${ }^{26} 2833$

In some respects, the most important findings involve comparisons between the two subgroups of patients who had no concurrent mental disorders. A concern about the desire for death in the context of euthanasia or assisted suicide requests is that depression and anxiety may result in cognitive distortions, perhaps transitory, that bias the decision to end one's life. ${ }^{3}$ Presumably, this bias is absent among those who have no particular mental health concerns. Another central finding, therefore, is that patients with a serious desire for death in the absence of a disorder still reported a higher degree of physical symptom distress and were more likely to endorse a sense of suffering. They were also more likely to acknowledge difficulties with social isolation and to feel like a burden to others. Interestingly, these social constructs figure prominently in a new 'interpersonal' theory of suicidal behavior, ${ }^{34}$ which apparently has some applicability to medical populations. ${ }^{35}$ The present findings suggest that it is perhaps relevant to the desire for death in the terminally ill as well.

Although we initially regarded a serious desire for death in the absence of a mental disorder as a 'manifestation of letting go' rather than an 'expression of despair', it appears that this desire is, nevertheless, embedded in a more complex clinical presentation. Thus, even when a formal mental disorder has been ruled out, a patient's report of a desire for death may serve as a catalyst to review physical symptom management, interpersonal concerns, and the overall sense of suffering.

There are several limitations to the study that must be noted. First, it was conducted as a secondary analysis of an existing dataset. Although the measures are appropriate to the purpose, dividing the sample into four subgroups, each with or without significant symptoms and concerns, resulted in small numbers of participants for some comparisons. Moreover, all the patients were receiving palliative care for cancer, and the prevalence of mental disorders and the desire for death may be different in other patient groups. A third limitation is that the study addresses patients' experience of the desire for death rather than direct requests for euthanasia or assisted suicide. Although participants in the NPCS were asked about their interest in receiving a physicianhastened death, only 22 individuals reported a readiness to end their lives in this way. ${ }^{24}$ This group was considered too small to subdivide on the basis of mental disorders. As we reported elsewhere, most (83\%) patients with an interest in receiving euthanasia also had a serious desire for death. Conversely, however, only 39\% of those with a serious desire for death would have opted for euthanasia, at least at the time of the interview. Thus, the desire for death and the desire for euthanasia are not synonymous, ${ }^{1}{ }^{36}$ and it is possible that they have differing associations with mental disorders.

Levene and Parker ${ }^{37}$ reviewed 21 studies pertaining to depression among people who have made explicit requests for euthanasia or assisted suicide. They concluded that in The Netherlands and the US State of Oregon, which permit these practices in some circumstances, $8-47 \%$ of patients who make such requests have high levels of depression. It is unclear, however, whether this rate is elevated in comparison with other patients with terminal illness. Moreover, even when depression is present, it is uncertain whether it is actually critical in either motivating the requests or in rendering the patients incompetent to make them. In The Netherlands, it has been found consistently that depressed patients are more likely to have their requests refused, ${ }^{38-40}$ suggesting that practitioners do appreciate the role of potentially treatable psychological problems.

There may be a paradox, however. Jurisdictions that permit euthanasia or physician-assisted suicide usually justify these practices as a way of providing relief from 'unbearable suffering'. ${ }^{41} 42$ Our results suggest that the experience of suffering is much more prevalent among those terminally ill individuals who have concurrent psychological disorders; yet in The Netherlands, these patients are apparently less likely to have their requests granted. Perhaps there is considerable societal acceptance of euthanasia as a way to assist patients in letting go at the end of life, but more hesitation when it is used to relieve their expression of despair. It may also be that healthcare providers make a distinction between despair that may or may not be remediable. Although these two pathways to the desire for death are distinct conceptually, in practice they may not be mutually exclusive.

\section{Author affiliations}

${ }^{1}$ Department of Psychology, The Ottawa Hospital

Rehabilitation Centre, Ottawa, Ontario, Canada

${ }^{2}$ Department Psychology, University of Ottawa,

Ottawa, Ontario, Canada 
${ }^{3}$ Department of Psychiatry, University of Manitoba, Winnipeg, Manitoba, Canada

${ }^{4}$ Department of Oncology, University of Calgary, Calgary, Alberta, Canada

${ }^{5}$ Faculty of Pharmacy, Université Laval, Québec, Québec, Canada

${ }^{6}$ Grey Nuns Community Hospital, Covenant Health, Edmonton, Alberta, Canada

${ }^{7}$ Department of Psychiatry, University of British Columbia-Okanagan, Kelowna, British Columbia, Canada

${ }^{8}$ Dr H. Bliss Murphy Cancer Centre, St. John's, Newfoundland and Labrador, Canada

${ }^{9}$ Department of Family Practice, University of British Columbia, Vancouver, British Columbia, Canada ${ }^{10}$ Division of Palliative Care Medicine, Department of Oncology, University of Alberta, Edmonton, Alberta, Canada

Acknowledgements The authors would like to acknowledge the contribution of the late Dr Pierre Allard, who collaborated in the study design and data collection.

Contributors KGW designed the study, obtained ethics approval, conducted analyses, drafted the paper, and is guarantor. TLD undertook data cleaning and analyses, and drafted the paper. HMC designed the study, obtained ethics approval, collected data, and revised the draft paper. SC, PRG, KM, MDL, FOS, DK, and RLF obtained ethics approval, collected data, and revised the draft paper.

Funding The Canadian National Palliative Care Survey was supported by a grant (reference no. 42538) from the Canadian Institutes of Health Research (CIHR). HMC is supported by a Tier 1 Canada Research Chair of the CIHR.

\section{Competing interests None.}

Ethics approval Ottawa Hospital Research Ethics Board.

Provenance and peer review Not commissioned; externally peer reviewed.

\section{REFERENCES}

1 Hudson PL, Kristjanson LJ, Ashby M, et al. Desire for hastened death in patients with advanced disease and the evidence base of clinical guidelines: a systemic review. Palliat Med 2006;20:693-701.

2 Breitbart W, Rosenfeld B, Pessin H, et al. Depression, hopelessness, and desire for hastened death in terminally ill patients with cancer. JAMA 2000;282:2907-11.

3 Chochinov HM, Wilson KG, Enns M, et al. Desire for death in the terminally ill. Am J Psychiatry 1995;152:1185-91.

4 Kelly BJ, Burnett P, Pelusi D, et al. Terminally ill cancer patients' wish to hasten death. Palliat Med 2002;16:339-45.

5 Kelly BJ, Burnett PC, Pelusi D, et al. Association between clinician factors and a patient's wish to hasten death: terminally ill cancer patients and their doctors. Psychosomatics 2004;45:311-18.

6 Kelly BJ, Pelusi D, Burnett PC, et al. The prevalence of psychiatric disorder and the wish to hasten death among terminally ill cancer patients. Palliat Support Care 2004;2:163-9.

7 O'Mahony S, Goulet J, Kornblith A, et al. Desire for hastened death, cancer pain and depression: report of a longitudinal observational study. J Pain Symptom Manage 2005;29:446-57.

8 Price A, Lee W, Goodwin L, et al. Prevalence, course and associations of desire for hastened death in a UK palliative population: a cross-sectional study. BMJ Support Palliat Care 2011;1:140-8.

9 Rosenfeld B, Breitbart W, Galietta M, et al. The Schedule of Attitudes toward Hastened Death: measuring desire for death in terminally ill cancer patients. Cancer 2000;88:2868-75.

10 Julião M, Barbosa A, Oliveira F, et al. Prevalence and factors associated with desire for death in patients with advanced disease: results from a Portuguese cross-sectional study. Psychosomatics 2013;54:451-7.

11 Kelly BJ, Burnett PC, Pelusi D, et al. Factors associated with the wish to hasten death: a study of patients with terminal illness. Psychol Med 2003;33:75-81.

12 Mystakidou K, Rosenfeld B, Prapa E, et al. Desire for death near the end of life: the role of depression, anxiety and pain. Gen Hosp Psychiatry 2005;27:258-62.

13 Mystakidou K, Parpa E, Katsouda E, et al. Influence of pain and quality of life on desire for hastened death in patients with advanced cancer. Int J Palliat Nurs 2004;10:476-83.

14 Rodin G, Zimmermann C, Rudall A, et al. The desire for hastened death in patients with metastatic cancer. J Pain Symptom Manage 2007;33:661-75.

15 Mystakidou K, Rosenfeld B, Prapa E, et al. The Schedule of Attitudes Toward Hastened Death: validation analysis in terminally ill cancer patients. Palliat Support Care 2004;2:395-402.

16 Mystakidou K, Prapa E, Tsilika E, et al. Depression, hopelessness, and sleep in cancer patients' desire for death. Int J Psychiatry Med 2007;37:201-11.

17 Jones JM, Huggins MA, Rydall AC, et al. Symptomatic distress, hopelessness, and the desire for hastened death in hospitalized cancer patients. J Psychosom Res 2003;55:411-18.

18 Rodin G, Lo C, Mikulincer M, et al. Pathways to distress: the multiple determinants of depression, hopelessness, and the desire for hastened death in metastatic cancer patients. Soc Sci Med 2009;68:562-9.

19 Rosenfeld B, Breitbart W, Gibson C, et al. Desire for hastened death among patients with advanced AIDS. Psychosomatics 2006;47:504-12.

20 McClain CS, Rosenfeld B, Breitbart W. Effect of spiritual well-being on end-of-life despair in terminally-ill cancer patients. Lancet 2003;361:1603-7.

21 Tiernan E, Casey P, O’Boyle C, et al. Relations between desire for early death, depressive symptoms and antidepressant prescribing in terminally ill patients with cancer. J R Soc Med 2002;95:386-90.

22 Brown JH, Henteleff P, Barakat S, et al. Is it normal for terminally ill patients to desire death? Am J Psychiatry 1986;143:208-11.

23 Nissim R, Gagliese L, Rodin G. The desire for hastened death in individuals with advanced cancer: a longitudinal qualitative study. Soc Sci Med 2009;69:165-71.

24 Wilson KG, Chochinov HM, McPherson CJ, et al. Desire for euthanasia or physician-assisted suicide in palliative cancer care. Health Psychol 2007;26:314-23.

25 Wilson KG, Chochinov HM, McPherson CJ, et al. Suffering with advanced cancer. J Clin Oncol 2007;25:1691-7.

26 Wilson KG, Chochinov HM, Skirko MG, et al. Depression and anxiety disorders in palliative cancer care. J Pain Symptom Manage 2007;33:118-29.

27 Wilson KG, Graham ID, Viola RA, et al. Structured interview assessment of symptoms and concerns in palliative care. Can J Psychiatry 2004;49:350-8.

28 Thompson GN, Chochinov HM, Wilson KG, et al. Prognostic acceptance and the well-being of patients receiving palliative care for cancer. J Clin Oncol 2009;27:5757-62. 
29 Wilson KG, Chochinov HM, Allard P, et al. Prevalence and correlates of pain in the Canadian National Palliative Care Survey. Pain Res Manag 2009;14:365-70.

30 Spitzer RL, Williams JBW, Kroenke K, et al. Utility of a new procedure for diagnosing mental disorders in primary care: the PRIME-MD 1000 study. JAMA 1994;272:1749-56.

31 American Psychiatric Association. Diagnostic and statistical manual of mental disorders. 4th edn. Washington, DC: American Psychiatric Association, 1994.

32 Chochinov HM, Wilson KG, Enns M, et al. Prevalence of depression in the terminally ill: effects of diagnostic criteria and symptom threshold judgments. Am J Psychiatry 1994;151:537-40.

33 Hallberg IR. Death and dying from old people's point of view a literature review. Aging Clin Exp Res 2004;16:87-103.

34 Van Orden KA, Witte TK, Cukrowicz KC, et al. The interpersonal theory of suicide. Psychol Rev 2010;117:575-600.

35 Wilson KG, Kowal J, Henderson PR, et al. Chronic pain and the interpersonal theory of suicide. Rehabil Psychol 2013;58:111-15.

36 Monforte-Royo C, Villavicencio-Chávez C, Tomás-Sábado J, et al. The wish to hasten death: a review of clinical studies. Psychooncology 2011;20:795-804.
37 Levene I, Parker M. Prevalence of depression in granted and refused requests for euthanasia and assisted suicide: a systemic review. J Med Ethics 2011;37:205-11.

38 Onwuteaka-Philipsen BD, Rurup ML, Pasman HR, et al. The last phase of life. Who requests and who receives euthanasia or physician-assisted suicide? Med Care 2010;48:596-603.

39 Jansen-van der Weide MC, Onwuteaka-Philipsen BD, Van Der Wal G. Granted, undecided, withdrawn, and refused requests for euthanasia and physician-assisted suicide. Arch Intern Med 2005;165:1698-704.

40 Haverkate I, Onwuteaka-Philipsen BD, van Der HA, et al. Refused and granted requests for euthanasia and assisted suicide in The Netherlands: interview study with structured questionnaire. BMJ 2000;321:865-6.

41 Dees M, Vernooij-Dassen M, Dekkers W, et al. Unbearable suffering of patients with a request for euthanasia or physician-assisted suicide: an integrative review. Psychooncology 2010;19:339-52.

42 Dees M, Vernooij-Dassen M, Dekkers W, et al. Unbearable suffering: a qualitative study on the perspectives of patients who request assistance in dying. J Med Ethics 2011;37:727-34. 